\title{
PUFA in the pathogenesis and treatment of patients with multiple sclerosis
}

\author{
L. S. Harbige ${ }^{1,2}$, E. Pinto ${ }^{1}$, M. Xiang ${ }^{1}$, M. Leach ${ }^{1,2}$ and M. K. Sharief ${ }^{3}$ \\ ${ }^{1}$ Centre for Biosciences Research, School of Science, University of Greenwich, Kent, UK, ${ }^{2}$ Medway School of Pharmacy, \\ Universities of Kent and Greenwich, Kent, UK and ${ }^{3}$ Department of Neurology, King's, Guy's and St Thomas' Hospital, \\ London, $U K$
}

Multiple sclerosis (MS) is a central nervous system-specific demyelinating disease, and most patients demonstrate a relapse-remitting form of the disease characterised by attacks (relapses) and periods of recovery (remission). Although the aetiology of MS remains unknown, much evidence suggests the presence of autoimmune mechanisms in the disease pathogenesis. Cytokine dysregulation has been found in peripheral blood mononuclear cells (PBMC) during the relapse-remitting phases of MS, i.e. raised TNF $\alpha$ and IL-1 $\beta$ and lack of regulatory transforming growth factor $\beta$ (TGF $\beta$ ) production ${ }^{(1)}$, and in an animal model of MS a protective effect of $\gamma$-linolenic acid (GLA; 18: $3 n-6)$-rich borage oil associated with raised TGF $\beta^{(2)}$. The aim of the present study was to determine the effects of a selected GLA-rich oil (BGC20-884) at two doses (a low dose $(5 \mathrm{~g} / \mathrm{d})$ and a high dose $(14 \mathrm{~g} / \mathrm{d})$ ) and a placebo control (PEGA) on the clinical course and PBMC cytokine and membrane fatty acid profiles of thirty-six patients with active MS in a randomized double-blind placebo-controlled trial format over 18 months. Patients were diagnosed and assessed using international criteria for MS. Relapse rate and expanded disability status scale (EDSS) were assessed every 3 months and blood taken and PBMC isolated for cytokine studies and membrane fatty acids.

High-dose BGC20-884 treatment markedly and significantly reduced the annualized mean of relapses per patient per year over 18 months (Figure) and disability progression (EDSS) compared with the placebo control and low-dose BGC20-884 treatment. PBMC cytokine changes ran parallel with the clinical findings, i.e. high-dose BGC20-884 showed no changes in TGF $\beta$ :TNF $\alpha$ and TGF $\beta$ :IL-1 $\beta$ and no changes in membrane fatty acids, whilst the placebo control and low-dose BGC20-884 group showed significant decreases in TGF $\beta$ :TNF $\alpha$ and TGF $\beta$ :IL- $1 \beta$ and loss of $n-6$ fatty acids, particularly linoleic acid (18: $2 n-6)$ and arachidonic acid (20: $4 n-6)$, over time. It is concluded that high-dose BGC20-884 markedly affects the clinical course of MS. Furthermore, the EDSS improvement in the highdose group suggests there maybe a beneficial effect on neuronal lipids and neural function in MS. The present study supports the hypothesis of dysregulation of fatty acid metabolism and cytokines in $\mathrm{MS}^{(1,2)}$.

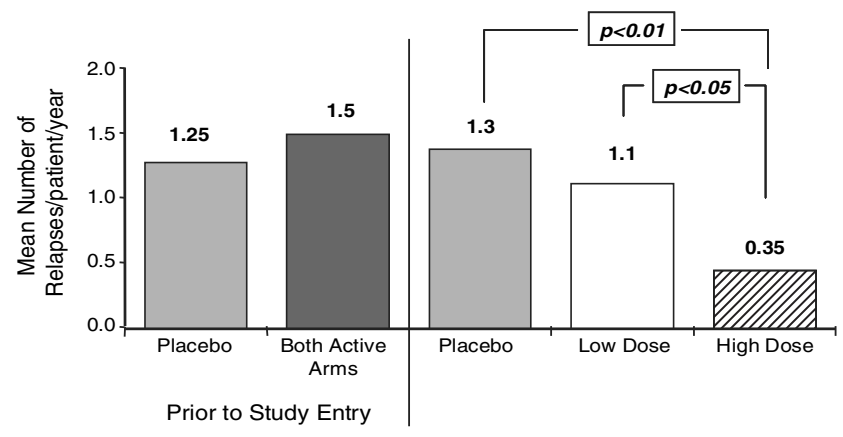

1. Hollifield, R, Harbige L, Pham-Dinh D \& Sharief MK (2003) Autoimmunity 36, 133-144.

2. Harbige L, Layward M, Morris-Downes M, Dumonde D \& Amor A (2000) Clin Exp Immunol 122, 445-452. 